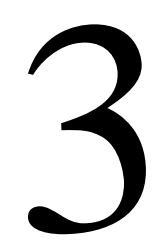

\title{
DERECHO A LA EDUCACIÓN DE LA INFANCIA Y DESIGUALDADES DE ORIGEN EN CENTROAMÉRICA
}

\section{Children's Right to Education and Social Background}

\author{
Inequalities in Central America
}

Miriam Lorente Rodríguez*

\section{$\underline{\text { RESUMEN }}$}

En este trabajo, abrazamos una comprensión de la infancia centroamericana y su derecho a la educación desde los enfoques de Derechos Humanos y de Capacidades de Sen y Nussbaum. Así, partiendo de las premisas de que niños, niñas y adolescentes son sujetos de derechos; la infancia constituye una etapa con identidad propia, clave en el desarrollo de las personas y la educación es un derecho humano fundamental, nos adentrarnos en el establecimiento y estudio de diversos escenarios educativos en los países centroamericanos y en el impacto de las desigualdades de origen en la configuración de las trayectorias escolares. Todo ello, con la finalidad de mostrar el panorama concreto de la subregión centroamericana en el camino hacia el cumplimiento real y efectivo del derecho a la educación de su infancia.

PAlabras Clave: Infancia, Derecho a la Educación, Capacidades, Desigualdades de Origen, Centroamérica.

\footnotetext{
* Universitat de València (España).
} 


\begin{abstract}
$\underline{\text { ABSTRACT }}$
In this paper, we embrace an understanding of Central American childhood and their right to education from the Human Rights and Capabilities approaches of Sen and Nussbaum and, as such, we start from the premise that children and adolescents are subjects of rights, childhood is a stage with its own identity-key in the development of people-, and education is a fundamental human right with this in mind we go into the establishment and study of several educational scenes in Central American countries and the impact of social background inequalities in the configuration of school careers. All this, in order to show a concrete panorama of the Central American region on its way to the real and effective implementation of the right to education from infancy.
\end{abstract}

KEY WORDS: Childhood, Right to Education, Capabilities, Social Background Inequalities, Central America.

$* * * * *$

\title{
1. INTRODUCCIÓN
}

El convencimiento de que la infancia ${ }^{1}$ es una etapa con identidad propia y fundamental en el desarrollo de las personas es un hecho ampliamente aceptado que ya acumula un vasto conocimiento en torno a la cuestión y que, desde diferentes enfoques, prueba las bondades del cultivo de una etapa trascendental en el desarrollo humano.

De este modo, la infancia constituye una etapa del desarrollo humano fundamental para el correcto desarrollo cognitivo, afectivo y social de la persona ya que se producen acelerados cambios madurativos y se desarrollan las capacidades y habilidades básicas de pensamiento y relación con el medio social y natural. Es por ello que el cuidado y la estimulación del niño y la niña durante la primera infancia son fundamentales.

No obstante, desde una perspectiva histórica, la concepción de los niños y niñas como adultos en miniatura perdurará durante siglos en la mentalidad colectiva social contribuyendo gravemente a la falta de un necesario reconocimiento y trato específico de la

\footnotetext{
${ }^{1}$ En este trabajo, siguiendo la Convención de los Derechos del Niño de 1989, "se entiende por niño todo ser humano menor de dieciocho años de edad, salvo que, en virtud de la ley que le sea aplicable, haya alcanzado antes la mayoría de edad" (art. 1). Siguiendo la definición de niño en el Sistema Interamericano de Derechos Humanos que, a su vez, se basa en la ofrecida por la Convención de Derechos del Niño, la Corte Interamericana precisó en la Opinión Consultiva 17 que el término niño "abarca, evidentemente, los niños, las niñas y adolescentes". Y respecto al concepto de primera infancia se sigue la definición realizada por el Comité de Derechos del Niño. Así pues, "en su examen de los derechos en la primera infancia, el Comité desea incluir a todos los niños pequeños: desde el nacimiento y primer año de vida, pasando por el período preescolar hasta la transición al período escolar. En consecuencia, el Comité propone como definición de trabajo adecuada de la primera infancia, el período comprendido hasta los 8 años de edad" (COMITÉ DE LOS DERECHOS DEL NIÑO, 2006).
} 
infancia. Circunstancia que no cambiaría hasta bien entrado el siglo XVIII, momento en el cual el trabajo de filósofos y pedagogos unido al de otros profesionales del área de la psicología y la medicina iluminaría el camino de una nueva ciencia, la paidología. Realidad que marcaría un punto de inflexión con el pasado generando un interés cada vez mayor por la infancia. De este modo, el análisis de la infancia desde las principales teorías sobre historia de la infancia se basan en los siguientes presupuestos, aun cuando han sido cuestionadas e incluso rechazas por otros autores (DÁVILA y NAYA, 2010): 1- Antes del siglo XVIII no hubo concepto de niñez; 2- Existió una relación formal entre padres e hijos marcada por la inferioridad de estos últimos y la distancia entre ellos; 3- Los niños fueron explotados brutalmente y sometidos a indignidades incluso hasta principios del siglo XIX.

Ahora bien, será el siglo XX aquel en el que, por excelencia, el reconocimiento de la infancia como sujeto de derecho y la búsqueda del mejoramiento y bienestar de ésta adquiera mayor esplendor, consenso y vigorosidad. Desarrollos y tendencias que siguen vigentes en el siglo XXI e informan el proceder actual. Así pues, hoy en día, no cabe duda que los derechos de la infancia son una temática de indudable interés en el seno de la comunidad internacional y, de forma muy especial, el derecho a la educación. Así lo pone de manifiesto todo un universo científico, político y legislativo creado ex profeso para el estudio, tratamiento específico y protección de la infancia y del derecho a la educación.

En coherencia con esta sucinta aproximación histórica puede evidenciarse que la noción de infancia es una construcción social. A este respecto:

"el niño pasa de ser una entidad biológica a una entidad social, por lo que, para completar teorías clásicas, hará falta entender la infancia como dicha construcción social, como una construcción histórica y cultural determinada" (ANCHETA, 2011: 20).

Por tanto, para poder comprender las diferentes visiones que se poseen de la infancia como construcción de las influencias sociales, es fundamental atender a la naturaleza socialmente construida de la misma; la invisibilidad del niño en la medida en que se continúan adoptando medidas en beneficio de éstos; y la necesidad de explorar las posibilidades de los niños como agentes activos que intervienen en la construcción de su propia historia (ANCHETA, 2011).

En este trabajo, a efectos de operativizar el análisis realizado, el referente principal relativo a la infancia será el establecido en la Convención sobre los Derechos del Niño de 1989. También, lo serán las aportaciones que realizan organismos internacionales que trabajan por y para el bienestar de la infancia. Así pues, bajo el amplio marco que ofrece la citada Convención recordamos aquí, por su carácter abierto y holístico, la siguiente definición: 
"la época en la que los niños y niñas tienen que estar en la escuela y en los lugares de recreo, crecer fuertes y seguros de sí mismos y recibir el amor y el estímulo de sus familias y de una comunidad amplia de adultos. Es una época valiosa en la que los niños y las niñas deben vivir sin miedo, seguros frente a la violencia, protegidos contra los malos tratos y la explotación. Como tal, la infancia significa mucho más que el tiempo que transcurre entre el nacimiento y la edad adulta. Se refiere al estado y la condición de la vida de un niño, a la calidad de esos años" (UNICEF, 2004)

Sea como fuere, se hace necesario en este punto tener presente que, independientemente de los matices diferenciales que unos y otros puedan aportar a la noción de infancia, la característica fundamental que subyace a todos ellos es el paso a una concepción de los niños, las niñas y adolescentes como sujetos de derechos.

\section{UNA APROXIMACIÓN A LA INFANCIA Y EL DERECHO A LA EDUCACIÓN DESDE LOS ENFOQUES DE DERECHOS HUMANOS Y DE CAPACIDADES}

Primeramente, se hace necesario matizar que la centralidad otorgada al derecho a la educación en nuestro trabajo, destacándolo de entre el resto de derechos de la infancia, se fundamenta en la consideración de que su garantía permite a las personas apoderarse de otra serie de derechos que también son básicos para su desarrollo y bienestar (keyright).

En lo que concierne al tratamiento educativo de la infancia, existen en la actualidad tres posicionamientos. El primero de ellos, desde una perspectiva económica, considera la educación y la infancia como una inversión que producirá beneficios en el futuro y reducirá costos que serían muy superiores a la inversión realizada derivados de los abandonos, la deserción y el bajo aprovechamiento de unos recursos humanos futuros que se encuentran relacionados con la falta de atención y educación de la infancia desde una temprana edad. El segundo, considera que la infancia es una etapa que hay que estimular al máximo para potenciar las capacidades de los seres humanos. En todo caso, desde los planteamientos hasta ahora explicados, la infancia no tiene una entidad propia en sí misma, sino que es considerada como una etapa de transición y que es necesaria ejercitar para extraer los máximos beneficios del adulto de mañana. Sin embargo, en el tercer posicionamiento, desde el enfoque de Derechos Humanos en el cual nos situamos en este estudio, el niño es concebido como sujeto de derechos y, por tanto, la infancia es una etapa propia con finalidades intrínsecas e inherentes a la condición de ser niño y niña.

A continuación, haremos mención a los instrumentos esenciales que incardinan el enfoque de Derechos Humanos de entre las múltiples declaraciones, tratados, acuerdos y convenciones que de un modo más o menos directo versan sobre nuestra temática de estudio. La Declaración Universal de Derechos Humanos del año 1948 es origen del movimiento contemporáneo internacional de derechos humanos y forma parte del Sistema Universal de Derechos Humanos que interacciona, en lo que a nuestro ámbito de estudio concierne, con la Declaración Americana sobre Derechos Humanos del año 1948 y la 
Convención Americana sobre Derechos Humanos del año 1969, que forman parte del Sistema Americano de Derechos Humanos. Y, adoptada por ambos sistemas, por su tratamiento integral y prolífico de los derechos relativos a la infancia, con un amplio consenso mundial conseguido en torno a ella convirtiéndola en el principal referente internacional para la infancia en materia de Derechos Humanos: la Convención sobre los Derechos del Niño del año 1989.

Desde la ratificación de la Convención sobre los Derechos del Niño por los países latinoamericanos se ha producido un aumento sustancial de la producción legislativa en materia de infancia mediante planes, agendas y leyes muy variadas; un proceso de adecuación de la propia legislación nacional a los compromisos internacionales adoptados en dicha Convención que se refleja, principalmente, en los Códigos de la Niñez y la Adolescencia que poseen la mayoría de países de la región latinoamericana; así como cambios fundamentales en las políticas sociales en defensa de los derechos de los niños, niñas y adolescentes, marcando todo ello un nuevo periodo en la consideración de éstos como sujetos de derechos.

Coherentemente, ha sido creada toda una red nacional, regional e internacional de instituciones que se encargan de velar por el cumplimiento de dichos derechos a muy distintos niveles de decisión e intervención entre los cuales destacamos como el más importante, dentro del Sistema Universal de Derechos Humanos, al Comité de los Derechos de Niño que supervisa la aplicación de los Estados Partes de la Convención de los Derechos del Niño. En la subregión centroamericana, guiada por el Sistema Interamericano de Derechos Humanos, hemos de señalar la labor de jurisprudencia de la Comisión y la Corte Interamericana de Derechos Humanos que, en materia de derechos de la infancia, se complementa con el Sistema Universal de Derechos Humanos en los ámbitos de desarrollo sustantivo, de la prueba y del monitoreo y evaluación de situaciones generales. En lo que a la relación específica con el Comité de los Derechos del Niño se refiere, las observaciones finales sobre situaciones de países que emite éste sirven de referente para la Comisión en lo que respecta a la evaluación de las situaciones de los derechos humanos en la región. Del mismo modo que las observaciones finales también pueden servir de prueba sobre la violación de derechos humanos en casos individuales ante la Corte (OEA-CIDH, 2009).

Así pues, existe una complementariedad entre los distintos sistemas de protección de derechos humanos de forma que se refuerzan mutuamente en el desarrollo de estándares internacionales en torno a estos. En este sentido, en materia de derechos de la infancia, la Corte Interamericana de Derechos Humanos estableció que la Convención Americana de Derechos Humanos y la Convención sobre los derechos del Niño forman parte de un corpus juris internacional de protección de los derechos de niños y niñas, lo que significa una 
conexión sustantiva entre ambos tratados y deben aplicarse de manera conjunta en los casos concernientes a la infancia. ${ }^{2}$

A continuación, se examinan las dos orientaciones clave para este estudio que se derivan del enfoque de Derechos Humanos, a saber, la del cambio de perspectiva en la concepción jurídica de la infancia que pasa a ser considerada sujeto de derechos, fundamentalmente a partir de la Convención sobre los Derechos del Niño y la de la importancia central del derecho a la educación, como hemos mencionado con anterioridad, también presente en el enfoque de Capacidades.

El enfoque de Derechos Humanos, a partir de la Convención de los Derechos del Niño supone para la infancia un cambio trascendental con respecto a su situación hasta entonces. Los niños gozan de derechos establecidos legalmente los cuales pueden ser exigidos; estos derechos son universales e inalienables frente al relativismo cultural; son indivisibles e iguales en importancia frente a jerarquizaciones que no reconocen la integralidad de la infancia; los niños pasan de ser beneficiarios pasivos a participantes activos (muy relacionado con la importancia de la agencia en la teoría de Capacidades de Sen); y, por tanto, desembocando ya en el punto clave donde nos vamos a detener, los niños se conciben como sujetos de derechos y no como objetos de protección, lástima y caridad.

Dentro del contexto latinoamericano este aspecto es fundamental pues, sin excepción alguna, el tratamiento legal de los menores en la región y en la subregión centroamericana hasta la promulgación de la Convención de los Derechos del Niño se ha basado en la doctrina de la Situación Irregular frente al nuevo paradigma que emerge de la Convención, el paradigma del Desarrollo Integral o doctrina de la Protección Integral. Cambio de concepción trascendental que vamos a revelar sucintamente de la mano de Salazar (2005).

A este respecto, la doctrina de la Situación Irregular, imperante en el tratamiento legal de la infancia durante los dos últimos siglos, básicamente consideraba a los menores de edad como objetos de protección por parte del Estado. Sólo actuaba ante aquella población infantil que no tenía satisfecha sus necesidades básicas, eran abandonados, inadaptados o infractores (a la que se le denominó menores) frente a aquella otra que sí tenían satisfechas sus necesidades y no eran objeto de intervención o castigo alguno (a la que se le denominó niños y niñas). Los primeros eran tratados como objeto de caridad, de compasión y criminalizados ante la justicia cuando cometían algún delito, legitimando esta última su proceder en relación a los menores de forma indiscriminada.

\footnotetext{
${ }^{2}$ Esta noción ha sido aplicada por la Corte en reiteradas ocasiones, como por ejemplo, en el caso Villagrán Morales y otros ("Niños de la Calle") contra Guatemala. Sentencia de 19 de noviembre de 1999. Serie C. No63, párrafo 194 (OEA-CIDH, 2009).
} 
Frente a este enfoque judicializado, tutelar y proteccionista en el que no existe nadie que, potencialmente, no pueda ser declarado irregular se abre paso la doctrina de la protección integral en los años noventa del siglo XX estimulada por el nacimiento de la Convención sobre los Derechos del Niño. Desde este nuevo paradigma, que es el que defendemos en nuestro estudio, se aboga por un abordaje integral de la infancia y no sólo judicial; ya no hay dos tipos de infancia claramente diferenciados, sino un universo integrado de niñez y adolescencia que son sujetos de derechos y no objetos de compasión y, a su vez, represión; y finalmente, al amparo del interés superior del niño, se entiende que el Estado debe dejar de ejercer ese papel tutelar y convertirse en promotor del bienestar y desarrollo integral de la infancia. No obstante, aunque es este último modelo el que camina hacia el ideal deseado, el trabajo de adecuación legislativa latinoamericana todavía carga con elementos de la doctrina de la Situación Irregular, así como el talante y quehacer político en materia de infancia.

El otro factor crucial en nuestro estudio que nos permite vincular el enfoque de Derechos Humanos con el de Capacidades es el papel central de la educación como posibilitador de otros derechos, del desarrollo humano $\mathrm{y}$, en definitiva, del bienestar infantil. Conceptos estos dos últimos que compartimos en este estudio, donde se parte de una visión holística e integral de los derechos, con la visión ofrecida por Sen y Nussbaum en su teoría de las Capacidades.

Existen variadas divergencias y matizaciones entre el trabajo de Sen (2000) y el de Nussbaum (2011) en torno al enfoque de Capacidades, relacionadas principalmente con la elaboración del listado de capacidades que Nussbaum si realizó y Sen orientó y dejó entrever algunas de ellas pero no las concretó. Decisiones las de ambos autores íntimamente vinculadas con la finalidad u orientación que cada uno quiso dar a las capacidades y al enfoque, en general. De este modo, la idea de capacidad puesta al servicio de la comparación entre países que ofrecía Sen no exigía una especificación de las capacidades, mientras que el carácter normativo del trabajo de Nussbaum en su pretensión de ofrecer una teoría de la democracia y de la justicia social básica, hacía de esta cuestión una tarea ineludible.

Sin embargo, a pesar de las diferentes inclinaciones intelectuales que han llevado a Sen y Nussbaum a desarrollar distintos aspectos, el enfoque es el mismo y, por tanto, las nociones y conceptos generales que son de nuestro interés son compartidos. A este respecto, el desarrollo humano se concibe como un proceso integrado de expansión de las libertades que disfrutan los individuos, relacionadas entre sí (SEN, 2000). En este último sentido se manifiesta Nussbaum al afirmar que "todos los elementos empleados en la teoría se entienden interconectados entre sí y que, como tales, se explican y esclarecen mutuamente" (NUSSBAUM, 2011:49). El vínculo relacional entre las distintas capacidades es clave en nuestro estudio y justifica el carácter holístico e integral del mismo puesto que partimos de la base de que, para garantizar el derecho a la educación en la infancia de 
forma efectiva, se requiere poseer libertad en otros aspectos claves para el desarrollo de las personas, lo que:

“exige la eliminación de las principales fuentes de privación de libertad: la pobreza y la tiranía, la escasez de oportunidades económicas y las privaciones sociales sistemáticas, el abandono en que pueden encontrarse los servicios públicos y la intolerancia o el exceso de intervención de los Estados represivos" (SEN, 2000: 19-20).

La importancia central que se le otorga al derecho a la educación se basa en que la consecución y ejercicio continuado del derecho a la educación será clave para potenciar el resto de derechos y se convertirá en un factor de protección de los mismos frente a esas fuentes de privación de libertad. En este sentido, Nussbaum (2011) reconoce que:

"la importancia de la educación ha sido un elemento central del enfoque de las capacidades desde sus comienzos (...) Ejerce asimismo una función capital para el desarrollo y la ejercitación de otras muchas capacidades humanas, es pues un `funcionamiento fértil’ de suma importancia para abordar los problemas de la desventaja y la desigualdad” (NUSSBAUM, 2011:181).

Y a diferencia de la manifestación de la autora a lo largo de su obra, -lo que se debe exigir a las personas son capacidades y no funcionamientos concretos,- cuando hace referencia a la educación en la infancia reconoce la exigencia de funcionamientos concretos por parte del Estado como, por ejemplo, la obligatoriedad de la misma. Esto es así por la inmadurez de niños y niñas y por "la espectacular expansión de capacidades que propicia en momentos posteriores de la vida" (NUSSBAUM, 2011: 185).

Por su parte, Sen (2000) también concibe la educación como una parte fundamental del desarrollo. La educación no sólo tiene un potencial instrumental, como así ha sido mantenido en la mayoría de teorías del desarrollo, sino también intrínseco en la medida que ésta es concebida como generadora de capacidades y, por tanto, de libertad. Por ello, junto con la sanidad y la igualdad de género, la educación ha sido contenido central en el trabajo de Sen. Muestra importante de este pensamiento se ha visto materializada en el Índice de Desarrollo Humano (IDH) con el que trabaja el PNUD.

Por tanto, a la luz de lo expuesto, baste decir que de sobra han sido probadas, en la literatura académica, las bondades de la educación para mitigar las grandes lacras que aquejan a las sociedades. Y, sin ser la pretensión ni objeto de este trabajo hacer un examen exhaustivo y prolífico de los beneficios de la educación ${ }^{3}$, simplemente, finalizar este epígrafe destacando que una educación proyectada con miras al desarrollo de los pueblos permite conciliar la enseñanza de una vida democrática, -que aumente las posibilidades de participación activa en la sociedad,- con la equidad y el crecimiento. Tiene el potencial de reducir las grandes desigualdades que aquejan a las sociedades latinoamericanas y es factor indiscutible en la lucha contra la pobreza y el analfabetismo, fuentes principales de

${ }^{3}$ Un análisis en profundidad de los beneficios no monetarios de la educación lo encontramos en Vila Lladosa, L.E. (2003). 
privación de capacidades básicas que no hacen sino negar a las personas el ejercicio de sus derechos más elementales y las oportunidades para desarrollarse y adquirir nuevas cotas de bienestar.

\section{DERECHO A LA EDUCACIÓN Y DESIGUALDADES DE ORIGEN EN LA ESCOLARIZACIÓN EN PRIMARIA Y SECUNDARIA EN CENTROAMÉRICA}

El derecho a la educación es un derecho humano fundamental y en nuestro estudio ocupa un lugar privilegiado precisamente por las enormes potencialidades que posee para posibilitar el ejercicio de otros derechos. Postura que hemos justificado. Por ello, es esencial garantizar unas condiciones óptimas de bienestar a la infancia que le permitan no sólo acceder a la educación sino permanecer y concluir con ciertas garantías niveles progresivos de escolaridad. Porque sólo así, la educación puede desplegar al máximo sus potencialidades y actuar como un factor fundamental de protección de la infancia.

Sin embargo, es posible advertir cómo en Centroamérica y, en general, en el conjunto de la región latinoamericana, la educación no consigue transformarse en un potente instrumento de igualación de oportunidades. En este sentido, SITEAL (2010) señala:

"es evidente que la profunda desigualdad en la distribución de los recursos socioculturales que caracteriza a América Latina encuentra su correlato en la configuración efectiva de las trayectorias escolares de sus niños y jóvenes, lo que permite reconocer, una vez más, que la escuela no es capaz de revertir las desventajas sociales de origen" (SITEAL, 2010:35).

A este respecto, va a analizarse, comparativamente, el panorama concreto que presenta la subregión centroamericana en el camino hacia el cumplimiento real y efectivo del derecho a la educación de su niñez y adolescencia. Para ello, se realiza una clasificación por grupos de países en función del nivel de conclusión de la educación primaria y secundaria que da lugar a tres escenarios educativos diferentes en la subregión, dentro de los cuales, se estudia la influencia de las variables socioeconómica (ingresos), sexo, geográfica, origen étnico y clima educativo del hogar en la configuración de las trayectorias escolares de los estudiantes. Además, este estudio se complementa con la proporción de niños y adolescentes que abandonaron la escuela o ni tan siquiera llegaron a asistir a la misma. 
Tabla 1. Escenario educativo de los países en función del nivel de conclusión primario y secundario combinados*. Influencia de las variables socioeconómica (ingresos), sexo, geográfica, origen étnico y clima educativo del hogar en las trayectorias escolares**. Niños con privaciones moderadas y graves en acceso a la educación ${ }^{* * *}$.

Centroamérica, alrededor de 2008.

\begin{tabular}{|c|c|c|c|}
\hline País & $\begin{array}{l}\text { Escenario } \\
\text { educativo }\end{array}$ & $\begin{array}{c}\text { Influencia de la variable nivel de ingresos, sexo, geográfica, } \\
\text { origen étnico y clima educativo del hogar }\end{array}$ & $\begin{array}{c}\text { Niños con } \\
\text { privaciones }\end{array}$ \\
\hline Costa Rica & \multirow{2}{*}{$\begin{array}{l}\text { Grupo A: } \\
\text { Egreso } \\
\text { alto } \\
\text { primaria y } \\
\text { medio } \\
\text { secundaria }\end{array}$} & \multirow{2}{*}{$\begin{array}{l}\text { - No hay brechas significativas en la asistencia al nivel primario. } \\
\text { En la conclusión del mismo, mayor incidencia del estrato social } \\
\text { que por área geográfica. Más marcadas en Panamá que en Costa } \\
\text { Rica, pero menor que en los grupos restantes. } \\
\text { - La variable sexo no muestra brechas significativas en el nivel } \\
\text { primario y el clima educativo del hogar revela leves diferencias de } \\
\text { hasta } 4 \text { puntos porcentuales entre hogares con bajo y alto clima } \\
\text { educativo, favorables a estos últimos. } \\
\text { - En el nivel secundario las brechas educativas son más marcadas } \\
\text { que en el nivel primario. Y muy superiores en la conclusión del } \\
\text { nivel secundario frente a la asistencia al mismo. Mayor incidencia } \\
\text { de las brechas educativas en la conclusión por estrato social que } \\
\text { por área geográfica. } \\
\text { - En Panamá las desigualdades de origen étnico no son marcadas } \\
\text { en la asistencia al nivel primario. Sin embargo, a medida que se } \\
\text { progresa en el sistema educativo éstas se profundizan de modo } \\
\text { que, en la asistencia y conclusión del nivel secundario, las } \\
\text { desigualdades son las más amplias registradas de todos los países } \\
\text { en detrimento de la población indígena. } \\
\text { - El capital cultural se muestra determinante en el acceso al nivel } \\
\text { secundario, con diferencias promedio de en torno a } 40 \text { puntos } \\
\text { porcentuales entre hogares con bajo y alto clima educativo, } \\
\text { favorables a estos últimos. }\end{array}$} & 6,2 \\
\hline Panamá & & & 6,3 \\
\hline País & $\begin{array}{l}\text { Escenario } \\
\text { educativo }\end{array}$ & $\begin{array}{l}\text { Influencia de la variable nivel de ingresos, sexo, geográfica, } \\
\text { origen étnico y clima educativo del hogar }\end{array}$ & $\begin{array}{l}\text { Niños con } \\
\text { privaciones }\end{array}$ \\
\hline El Salvador & $\begin{array}{l}\text { Grupo B: } \\
\text { Egreso } \\
\text { medio } \\
\text { primaria y } \\
\text { egreso } \\
\text { medio } \\
\text { secundaria }\end{array}$ & $\begin{array}{l}\text { - Brechas socioeconómicas y geográficas muy marcadas. Más en } \\
\text { la conclusión de los niveles educativos que en la asistencia. Y } \\
\text { más fuertes en el nivel secundario que en el nivel primario. } \\
\text { - Mayor incidencia de las brechas educativas por estrato social } \\
\text { que por área geográfica. } \\
\text { - La variable sexo establece leves diferencias favorables a las } \\
\text { mujeres en la conclusión primaria y más marcadas en la } \\
\text { asistencia al nivel secundario favorables a los varones. } \\
\text { - No hay desigualdad en función del origen étnico en detrimento } \\
\text { de la población indígena. Más bien asisten y concluyen en mayor } \\
\text { medida que sus pares mestizos y blancos. } \\
\text { - Las brechas en el acceso en función del clima educativo del } \\
\text { hogar son muy leves en el nivel primario, mientras que en el } \\
\text { nivel secundario son muy marcadas con diferencias de } 35 \text { puntos } \\
\text { porcentuales entre hogares con bajo y alto clima educativo } \\
\text { familiar, favorables a estos últimos. }\end{array}$ & 8,9 \\
\hline País & $\begin{array}{l}\text { Escenario } \\
\text { educativo }\end{array}$ & $\begin{array}{l}\text { Influencia de la variable nivel de ingresos, sexo, geográfica, } \\
\text { origen étnico y clima educativo del hogar }\end{array}$ & $\begin{array}{l}\text { Niños con } \\
\text { privaciones }\end{array}$ \\
\hline Guatemala & Grupo $C$ : & - Mayor incidencia de las brechas educativas por estrato social & 13,1 \\
\hline
\end{tabular}




\begin{tabular}{|c|c|c|c|}
\hline & \multirow[t]{3}{*}{$\begin{array}{l}\text { Egreso } \\
\text { medio } \\
\text { primaria y } \\
\text { egreso } \\
\text { bajo } \\
\text { secundaria }\end{array}$} & \multirow{3}{*}{$\begin{array}{l}\text { que por área geográfica. Son las más severas de todos los grupos } \\
\text { de países. Con algunas excepciones en las que El Salvador } \\
\text { posee niveles similares. } \\
\text { - La variable sexo no ejerce influencia, exclusivamente, en la } \\
\text { asistencia al nivel primario. No así en la asistencia al nivel } \\
\text { secundario que se muestra favorable a los varones y en la } \\
\text { conclusión de ambos niveles, favorable a las mujeres, excepto en } \\
\text { el caso guatemalteco. } \\
\text { - Las brechas son muy marcadas en la conclusión primaria pero } \\
\text { mucho más en la conclusión secundaria alcanzado promedios de } \\
\text { un } 60 \% \text { de diferencia entre quintiles de ingreso y del } 30 \% \text { en } \\
\text { detrimento de los residentes en áreas rurales. } \\
\text { - En Guatemala y Nicaragua la asistencia al nivel primario no } \\
\text { presenta brechas marcadas en función del origen étnico, incluso } \\
\text { en Nicaragua levemente favorables a la población indígena. La } \\
\text { conclusión de la primaria, así como el ingreso y conclusión } \\
\text { secundaria se muestra favorable a la población mestiza y blanca. } \\
\text { - La variable clima educativo del hogar muestra mínimas } \\
\text { diferencias en el nivel primario. Y en el caso de Honduras, a } \\
\text { tenor de la información que ofrece la base de datos de SITEAL, } \\
\text { esta diferencia es favorable a los hogares con bajo capital } \\
\text { cultural. En el nivel secundario las brechas son extremas, con } \\
\text { porcentajes diferenciales que van del } 30 \% \text { en Nicaragua al } 60 \% \\
\text { en Guatemala entre hogares con bajo y alto clima educativo } \\
\text { familiar, favorables a estos últimos. }\end{array}$} & \\
\hline Honduras & & & 13,1 \\
\hline Nicaragua & & & 11,6 \\
\hline
\end{tabular}

Fuente: Elaboración propia en base a SITEAL (2010): Informe sobre tendencias sociales y educativas en América Latina, 2010; SITEAL (2011a): Informe sobre tendencias sociales y Educativas 2011; SITEAL (2015): Base de datos (http://www.siteal.iipe-oei.org/base_de_datos/consulta); CEPAL (2010): Panorama social de América Latina 2010; CEPAL/UNICEF-TACRO (2010): Pobreza infantil en América Latina y El Caribe.

* La clasificación de los países es realizada por SITEAL (2010) y ha sido adaptada a los seis países objeto de este estudio.

** El análisis de las diferentes variables de origen es realizado a partir de CEPAL (2010), SITEAL (2011), SITEAL (2015). La influencia de las variables socioeconómica, de sexo y geográfica es analizada en la asistencia y conclusión del nivel primario y secundario de educación. El origen étnico en la asistencia y conclusión del nivel primario así como en el ingreso al nivel secundario de entre quienes finalizaron el nivel primario y la conclusión del nivel secundario. Y, finalmente, el clima educativo del hogar (bajo, medio y alto) en la escolarización neta de ambos niveles educativos.

*** Recoge la proporción de niños y adolescentes (de 6 a 17 años) que sufren privaciones graves (nunca asistieron a la escuela) o moderadas-graves (abandonaron la escuela).

La clasificación establecida en la Tabla 1 permite sintetizar la situación del derecho a la educación en los países centroamericanos en función del grado de conclusión del nivel primario y secundario de educación que las diferentes trayectorias o escenarios educativos de los países han permitido configurar.

Así pues, pueden clasificarse en tres grupos. El primero de ellos, conformado por Costa Rica y Panamá, es el que más cercano se encuentra al objetivo ideal de la universalización del acceso al conocimiento en la subregión centroamericana, es decir, que todos los niños puedan disfrutar del derecho a la educación y los beneficios que ésta puede reportarles, con un nivel alto de egreso primario y un egreso medio en el nivel secundario. 
El grupo segundo, representado por un único país, El Salvador, se encontraría en una posición intermedia más alejada del objetivo de lograr que el derecho a la educación llegue a todos que el primer grupo, con un egreso medio en el nivel primario y secundario, pero en una situación más favorable que el último grupo. Éste estaría integrado por Honduras, Guatemala y Nicaragua y constituyen el grupo de países que más lejos se encuentra de lograr que el derecho a la educación sea una realidad consumada, con un egreso medio en el nivel primario y bajo en el nivel secundario.

Haciendo una síntesis de las trayectorias escolares de los países centroamericanos, puede decirse, siguiendo a SITEAL (2010; 2011b) y CEPAL (2010), que en Costa Rica y Panamá la transición de primaria a secundaria se vuelve masiva a los 17 años. El problema principal que se plantea en Costa Rica es el abandono tardío, es decir, que después de haber tenido una asistencia masiva de niños a la escuela, en la adolescencia comienza un proceso de deserción y un porcentaje importante de alumnos abandona. Y sólo en la capital, San José, la asistencia educativa está generalizada (más del $80 \%$ de asistencia tanto en primaria como en secundaria). En Panamá, también se da con intensidad la problemática del abandono tardío en gran parte del país, pero el panorama es más heterogéneo con zonas de asistencia escolar generalizada y algunas zonas muy reducidas donde no se llega a generalizar el nivel (no se alcanza al 60\% de asistencia en ambos niveles educativos) o donde la incorporación escolar es tardía. Los niveles de repetición y deserción escolar en la educación primaria y secundaria de ambos Estados son los más bajos de los países centroamericanos, con porcentajes de alumnos rezagados dos años o más de en torno al $15 \%$ promedio y niveles de deserción del $25 \%$ en el recorrido escolar primario y secundario de jóvenes de 15 a 19 años.

Por su parte, en El Salvador los niños concluyen en menor medida el nivel primario que en Costa Rica y Panamá. A este respecto, la deserción durante el nivel primario es mayor que en estos dos países. También lo es el rezago educativo, de modo que una gran proporción de adolescentes permanecen en la educación primaria a edades avanzadas, lo que supone que a los 17 años no se ha completado aún el paso al nivel secundario. El abandono en este último es más moderado, de forma que los niveles de conclusión, si bien son más bajos, se aproximan a los del grupo anterior. Así pues, en términos generales el porcentaje de alumnos que se encuentran rezagados dos o más años tanto en el nivel primario como secundario asciende al 20,6\% y la proporción de deserción durante el ciclo escolar primario y secundario de jóvenes de 15 a 19 años asciende al 32,7\%. Cifras superiores a las del anterior grupo, como puede observarse, pero considerablemente más reducidas que las que reporta el último conjunto de países.

En Guatemala, Nicaragua y Honduras el rezago y la deserción educativa se dan más intensamente que en los grupos de países anteriores de forma que el porcentaje de alumnos con dos o más años de retraso escolar en primaria y secundaria, en promedio, al $32 \%$ de la 
población estudiantil. Y el porcentaje de alumnos de 15 a 19 años que abandonan el sistema educativo durante la escolaridad básica se sitúa en torno a un $42 \%$ en promedio. De este modo, el acceso a una trayectoria educativa esperada (satisfactoria) apenas tiene lugar. Sólo parece posible en las grandes ciudades o las capitales de estos países. Mientras tanto, en el resto de zonas de los mismos conviven diversas problemáticas en la configuración de estas trayectorias educativas, con lugares donde la incorporación tardía tiene una fuerte incidencia, o el abandono temprano y el tardío y otras zonas en las que no se generaliza la asistencia escolar a ninguna edad (es decir, ni en el nivel primario ni secundario de educación).

Así pues, claramente pueden diferenciarse estos tres grupos de países en función del cumplimiento con el derecho a la educación, los cuáles, presentan problemáticas y casuísticas particulares que son más severas en unos casos que en otros.

En coherencia con todo lo expresado, es en Guatemala, Honduras y Nicaragua donde la niñez y la adolescencia sufren las mayores privaciones en el acceso a la educación (nunca asistieron o abandonaron la escuela) con porcentajes que oscilan entre el 11,5\% en Nicaragua y el 13,1\% en Guatemala y Honduras. Por su parte, El Salvador se encontraría en una posición más favorable, pero no obstante, muy negativa con una proporción del $8,9 \%$ de niños privados del disfrute del derecho a la educación. La posición más favorable a este respecto la ostentan Costa Rica y Panamá con porcentajes de exclusión parejos al promedio latinoamericano, en torno al 6\%. No obstante, cualquier proporción es demasiada cuando ningún niño debiera estar excluido del sistema escolar. Por su parte, los países centroamericanos de Honduras, Nicaragua y Guatemala evidencian las peores cifras de toda Latinoamérica, junto con un único país suramericano, Perú (CEPAL/UNICEF-TACRO, 2010).

A continuación, va a analizarse el panorama que presentan los países objeto de estudio en relación con la desigualdad y exclusión que tiene lugar en el seno de los sistemas de educación centroamericanos. Las desigualdades educativas en función del nivel de ingresos son considerablemente superiores en todos los países a aquellas que se producen en función del lugar de residencia. En todo caso, se producen en detrimento de la población más empobrecida o residente en zonas rurales. Ambos tipos de segregación social tienen más influencia en la conclusión de los niveles educativos que en la asistencia y son más marcadas en el nivel secundario de educación que en el primario. En la asistencia de niños y niñas a este último, las variables socioeconómica y geográfica presentan brechas poco pronunciadas y el clima educativo del hogar y el origen étnico no generan diferencias significativas. Sin embargo, la conclusión del nivel primario de educación todavía revela diferencias muy marcadas en El Salvador, Honduras y, sobre todo, en Guatemala y Nicaragua en función del estrato social y el lugar de residencia. 
La variable sexo muestra también un patrón común en todos los países centroamericanos. El nivel de conclusión del nivel primario y secundario es favorable a las mujeres en todos los Estados, excepto en Guatemala donde se observan niveles ligeramente favorables a los varones, lo que puede estar relacionado con el papel asignado a la mujer en los grupos originarios ${ }^{4}$. En cambio, la asistencia a la enseñanza secundaria es superior entre los varones en todos los países, con brechas mucho más moderadas en Costa Rica y Panamá.

Así pues, el hecho de que los chicos concluyan en menor medida puede ser, en parte, explicado por la mayor incorporación de los varones a la fuerza de trabajo, como papel asignado tradicionalmente al varón. Las niñas muestran menor asistencia en todos los países al sistema de educación secundaria, lo que en parte puede guardar relación con los estereotipos sociales asignados a la mujer en la sociedad y la importancia social considerablemente menor atribuida a la educación de las niñas en niveles educativos superiores, especialmente en determinados colectivos, así como las dificultades añadidas que enfrentan las adolescentes a estas edades. En este sentido, la falta de servicios higiénicos apropiados en las escuelas puede ser un condicionante del acceso a la educación de las niñas durante la pubertad (UNESCO, 2007; ROMA, E. y PUGH, I., 2012). Sumado a la falta de comodidades o directamente situaciones discriminatorias que suelen padecer, en el seno de los sistemas educativos, las adolescentes embarazadas y madres jóvenes. Fenómeno, el del embarazo adolescente, de una magnitud muy considerable en la subregión centroamericana, alcanzando en países como Nicaragua y Honduras en torno al $25 \%$ y el $22 \%$ de las adolescentes (KHAN y VINOD, 2008). Por tanto, es fundamental emprender políticas gubernamentales de discriminación positiva que favorezcan la inclusión y permanencia en el sistema educativo de adolescentes embarazadas o madres; establezcan mecanismos para visibilizar, denunciar y erradicar posibles prácticas discriminatorias desde la institucionalidad de los centros educativos hacia este colectivo y, con rango universalista, que favorezcan la apropiación por parte de los y las adolescentes y jóvenes de sus derechos sexuales y reproductivos para que puedan ejercerlos de forma libre, sana y responsable ${ }^{5}$.

Como se ha comentado, las desigualdades sociales aumentan a medida que se progresa en el sistema educativo, de modo que en el nivel secundario las brechas socioeconómicas y geográficas son mucho más marcadas en todos los países,

\footnotetext{
4 Teniendo en consideración que alrededor del $40 \%$ de la población guatemalteca es indígena, resulta clarificador la aportación de CEPAL (2010) al respecto que explica que las jóvenes pertenecientes a pueblos originarios tienden a incorporarse más tempranamente al mercado de trabajo, por lo que muestran mayores frecuencias de abandono temprano y concluyen en menor medida.

5 Para profundizar más en la justificación de la necesidad de apropiarse de los derechos sexuales y reproductivos y cómo hacerlo desde la experiencia concreta costarricense, la obra editada por Muñoz y Ulate (2012) es de lectura ineludible. En ella se recogen ricas y variadas contribuciones desde diferentes sectores profesionales e ideológicos al programa de estudio "Educación para la afectividad y la sexualidad integral" del Ministerio de Educación Pública para apoyar y justificar la introducción del mismo en un sistema educativo sexista y patriarcal que lo había postergado en demasía.
} 
principalmente en la conclusión del mismo. A su vez, el clima educativo del hogar empieza a posicionarse como un factor condicionante en la escolarización de niños y niñas en el nivel secundario de educación y las brechas interétnicas se amplían considerablemente en detrimento de la población indígena para los países sobre los que se dispone de datos, a excepción de El Salvador. Si bien es cierto, en este país la población indígena es menor del $1 \%$ de la población total (DIRECCIÓN GENERAL DE ESTADÍSTICAS Y CENSOS DE EL SALVADOR, 2015).

Como se ha podido comprobar, las desigualdades socioeconómicas ejercen el mayor impacto en la configuración de las trayectorias educativas de la niñez y la adolescencia. No obstante, SITEAL (2008) explica que a medida que aumentan las edades, la tendencia de la desescolarización deja de ser situación exclusiva de la población estudiantil pobre y surgen con fuerza otros motivos del abandono escolar. En este mismo sentido se expresa el Programa Estado de la Nación (2011) cuando afirma que:

"Si bien la no asistencia escolar se explica fundamentalmente por las necesidades económicas de los niños, niñas y adolescentes y sus familias, conforme aumenta la edad otras justificaciones adquieren relevancia" (PROGRAMA DE ESTADO DE LA NACIÓN, 2011:376).

Así, a partir de los 12 años, pero sobre todo en el rango de edad de 15 a 17 años, la desescolarización se amplía entre los sectores sociales medios y altos y, a su vez, aspectos como la falta de motivación y el desinterés se convierten en razones importantes del abandono escolar a partir de los 15 años. De hecho, la falta de dinero y el desinterés son los dos principales motivos por los que los jóvenes de 15 a 17 años no asisten al nivel secundario (PROGRAMA ESTADO DE LA NACIÓN, 2011). Motivos que ayudan a comprender mejor el creciente impacto que el abandono tardío está teniendo en la subregión centroamericana y los bajos niveles de conclusión secundaria.

\section{CONSIDERACIONES FINALES}

A modo de cierre, destacar que las desigualdades de origen penetran en las estructuras educativas y se reproducen en éstas sin que el sistema educativo sea capaz de revertirlas al mismo tiempo que el propio sistema educativo, segmenta y estratifica en función de niveles muy diferentes de calidad y eficiencia educativa. De modo que los sectores sociales con menos recursos reciben una educación de menor calidad que sus pares con mayores recursos (CEPAL, 2010). Por lo que las desigualdades no sólo no se revierten, sino que tienden a ampliarse, sobre todo durante el nivel secundario.

En el estudio previo han podido advertirse diversos patrones o tendencias de desigualdad en los sistemas de educación centroamericanos. Así, las desigualdades socioeconómicas ejercen el mayor impacto en la configuración de las trayectorias educativas de la niñez y la adolescencia, por lo que el bienestar de las familias es un potente elemento condicionante del derecho a la educación. A este respecto, SITEAL (2007) 
explica que para que las familias latinoamericanas estén en condiciones de mantener un esfuerzo con la educación de los hijos que les permita permanecer al menos diez años en la escuela, se han de crear unas condiciones de bienestar e integración social ${ }^{6}$. No obstante, la realidad en la región es que el capital económico mínimo para una adecuada trayectoria educativa, junto con la valoración positiva de la educación son, al mismo tiempo, exponencialmente inalcanzables para un sector mayoritario.

A su vez, el impacto de las desigualdades de origen se incrementa conforme se progresa en el sistema educativo, de forma que en la educación secundaria las brechas socioeconómicas y geográficas son mucho más marcadas en todos los países, especialmente en la conclusión de este nivel educativo. Con respecto a la variable sexo, la tendencia existente revela que las niñas concluyen tanto la educación primaria como la secundaria en mayor medida que sus pares varones, salvo en Guatemala, mientras que la asistencia a la enseñanza secundaria es superior entre ellos. Asimismo, en el nivel secundario de educación, el clima educativo del hogar se convierte en un potente factor condicionante de la escolarización y las brechas interétnicas se amplían considerablemente.

Los diversos patrones de desigualdad que configuran las trayectorias educativas de la infancia centroamericana permiten concluir que los países de la subregión, sin excepción, están realizando esfuerzos considerables para lograr una enseñanza primaria universal para todos. Ahora bien, sin dejar de lado todo el camino que se lleva recorrido en el tramo primario de educación, ni las carencias aún existentes en el mismo, el examen realizado no nos permite sino incidir en la necesidad de reclamar un papel principal de la enseñanza secundaria en el marco de la política nacional de los respectivos países, ya que la conclusión de la misma es fundamental para asegurar un umbral de bienestar óptimo y poder romper con el círculo de la pobreza.

Finalmente, señalar que, en Centroamérica, los procesos de desigualdad y exclusión educativa van en detrimento de aquellos que residen en zonas rurales, pertenecen a hogares con un bajo nivel socioeconómico o un bajo capital cultural, o todo ello a la vez. Las capas de discriminación se van superponiendo y se retroalimentan unas a otras de tal modo que, en términos generales, ser indígena (y dentro ello bajo la condición de mujer), residir en zonas rurales y pertenecer a una familia con bajos ingresos y reducido capital cultural comprometen seriamente la posibilidad, real y efectiva, de disfrutar del derecho a la educación.

\footnotetext{
6 En este sentido, una serie de factores como la vivienda inadecuada, la carencia de infraestructura habitacional, los problemas de hacinamiento y el gran número de niños, los escasos recursos de capital humano, la fragilidad de los vínculos con el mercado laboral, así como la inestabilidad de los ingresos, entre otros se convierten en obstáculos al bienestar mínimo de las familias y a la asistencia regular a la escuela y la consecución de logros educativos (CEPAL, 2010).
} 


\section{REFERENCIAS BIBLIOGRÁFICAS}

ANCHETA ARRABAL, A. (2011): La escuela infantil hoy. Perspectivas internacionales de la educación y atención a la primera infancia (Valencia, Tirant Humanidades).

CEPAL (2010): Panorama social de América Latina 2010 (Santiago de Chile, CEPAL).

CEPAL/UNICEF-TACRO (2010): Pobreza infantil en América Latina y El Caribe (Santiago de Chile, CEPAL/UNICEF-TACRO).

COMITÉ DE LOS DERECHOS DEL NIÑO (2006): "Observación General $N^{o} 7$. Realización de los derechos del niño en la primera infancia”, $40^{\circ}$ período de sesiones, Ginebra, 12 a 30 de septiembre de 2005, Naciones Unidas, Convención de los Derechos del Niño /CRC/GC/7/ Rev.120, 20 de septiembre. Extraído de http://www2.ohchr.org/english/bodies/crc/docs/AdvanceVersions/GeneralComment 7Rev1_sp.pdf

DÁVILA, P. NAYA, L.M. (2010): El estudio de la infancia. Aproximación histórica y comparada. En P. DÁVILA y L. M. NAYA (Coords.), Infancia, Derechos y Educación en América Latina, pp.249-278 (Donostia, Espacio universitario erein).

DIRECCIÓN GENERAL DE ESTADÍSTICAS Y CENSOS DE EL SALVADOR (2015): Censo de población y vivienda 2007 (Población indígena). Extraído de http://www.digestyc.gob.sv/index.php/temas/des/poblacion-y-estadisticasdemograficas/censo-de-poblacion-yvivienda/población-censos.html

KHAN, S. \& VINOD, M. (2008): Youth Reproductive and Sexual Health. DHS Comparative Reports 19 (Calverton-Maryland, Macro International Inc).

MUÑOZ, V. Y ULATE, C. (eds.) (2012): El derecho humano a la educación para la afectividad y la sexualidad integral. Contribuciones para una reforma educativa necesaria (Heredia-C.R., UNA).

NACIONES UNIDAS (1989): Convención sobre los Derechos del Niño. Extraído de http://www.ohchr.org/SP/ProfessionalInterest/Pages/CRC.aspx

NUSSBAUM, M. (2011): Crear capacidades. Propuesta para el desarrollo humano (Barcelona, Paidós).

OEA-CIDH (2009): La infancia y sus derechos en el sistema interamericano de protección de derechos humanos (Washington D.C, OEA-CIDH).

PROGRAMA ESTADO DE LA NACIÓN (2011): Cuarto Informe Estado de la Región en Desarrollo Humano Sostenible (San José-C.R., PROGRAMA ESTADO DE LA NACIÓN).

ROMA, E. y PUGH, I. (2012): Toilets for health. A report by the London School of Hygiene and Tropical Medicine in collaboration with Domestos (Londres, London School of Hygiene and Tropical Medicine).

SALAZAR R.V. (2005): La niñez y sus derechos en el contexto costarricense (Heredia, C.R., CIDE-UNA).

SEN, A. (2000): Desarrollo y libertad (Barcelona, Planeta). 
SITEAL (2007): Informe sobre tendencias sociales y educativas en América Latina (Buenos Aires, IIPE-UNESCO-OEI).

SITEAL (2008): Informe sobre tendencias sociales y educativas en América Latina. La escuela y los adolescentes (Buenos Aires, IPE-UNESCO-OEI).

SITEAL (2010): Informe sobre tendencias sociales y educativas en América Latina. Metas educativas 2021: desafíos y oportunidades (Buenos Aires, IPE - UNESCO - OEI).

SITEAL (2011a): Informe sobre tendencias sociales y educativas en América Latina .La educación de los pueblos indígenas y afrodescendientes (Buenos Aires, IPEUNESCO-OEI)

SITEAL (2011b): Atlas de las desigualdades educativas en América Latina: La asistencia a la escuela en la actualidad. Distintas trayectorias educativas. Extraído de http://atlas.siteal.org/capitulo_3

SITEAL (2015): Base de datos. Extraído de http://www.siteal.iipeoei.org/base de datos/consulta

UNESCO (2007): Informe de seguimiento de la EPT en el mundo 2008. Educación para Todos en 2015 ¿Alcanzaremos la meta? (París, UNESCO).

UNICEF (2004): Estado mundial de la infancia 2005. La infancia amenazada (versión interactiva). Extraído de http://www.unicef.org/spanish/sowc05/

VILA LLADOSA, L.E. (2003): Los beneficios no monetarios de la educación, Revista de educación, 331, pp.309-324.

\section{PROFESIOGRAFÍA}

\section{Miriam Lorente Rodríguez}

Personal Investigador en el Departamento de Educación Comparada e Historia de la Educación de la Universidad de Valencia durante cuatro años. Profesora en el Máster Oficial en Acción Social Educativa de dicha universidad. Se licencia en Pedagogía, realiza un Máster Oficial en Política, Gestión y Dirección de Organizaciones Educativas y se doctora en Educación, en enero de 2014 en la Universidad de Valencia con la máxima calificación y menciones "Cum Laude" y "Doctor Internacional", obteniendo premios extraordinarios tanto de Licenciatura como de Máster. Las líneas de trabajo de investigación que desarrolla están relacionadas con los derechos de la infancia y su vinculación con el derecho a la educación en Latinoamérica, especialmente en Centroamérica desde el ámbito de la Educación Comparada; la relación entre la desigualdad de género, el subdesarrollo y la educación; la situación de la Educación Comparada en el ámbito nacional español tras la implementación del Espacio Europeo de Educación, su representación curricular y su impacto en la formación inicial de los profesionales de la Educación en España. Datos de contacto: Universidad de Valencia. Departamento de Educación Comparada e Historia de la Educación. Facultad de Filosofía y 
Ciencias de la Educación. Avenida Blasco Ibáñez no 30. (46010) Valencia. España. E-mail: Miriam.Lorente@uv.es

Fecha de recepción: 16 de julio de 2015.

Fecha de aceptación: 1 de febrero de 2016 